founding effects of age and dementia severity on cerebral metabolism in Alzheimer's disease. Hum. Brain Mapp. 10:39-48.

20. Aupee, A.M., et al. 2001. Voxel-based mapping of brain hypometabolism in permanent amnesia with PET. Neuroimage. 13:1164-1173.

21. Vogt, B.A., and Laureys, S. 2005. Posterior cingulate, precuneal and retrosplenial cortices: cytology and components of the neural network correlates of consciousness. Prog. Brain Res. 150:205-217.

22. Laureys, S., Lemaire, C., Maquet, P., Phillips, C., and Franck, G. 1999. Cerebral metabolism during vegetative state and after recovery to consciousness [letter]. J. Neurol. Neurosurg. Psychiatry. 67:121-122.

23. Laureys, S., Faymonville, M.E., Moonen, G., Luxen, A., and Maquet, P. 2000. PET scanning and neuronal loss in acute vegetative state. Lancet. 355:1825-1826.
24. Schiff, N.D. 2005. Modeling the minimally conscious state: measurements of brain function and therapeutic possibilities. Prog. Brain Res. 150:473-493.

25. Laureys, S., et al. 2000. Restoration of thalamocortical connectivity after recovery from persistent vegetative state. Lancet. 355:1790-1791.

26. Gould, E., Reeves, A.J., Graziano, M.S., and Gross, C.G. 1999. Neurogenesis in the neocortex of adult primates. Science. 286:548-552.

\title{
Arrhythmogenic right ventricular cardiomyopathy: moving toward mechanism
}

\author{
Calum A. MacRae, ${ }^{1}$ Walter Birchmeier, ${ }^{2}$ and Ludwig Thierfelder ${ }^{2,3}$ \\ ${ }^{1}$ Cardiovascular Research Center, Massachusetts General Hospital and Harvard Medical School, Boston, Massachusetts, USA. \\ 2Max Delbrück Center for Molecular Medicine, Berlin, Germany. ${ }^{3}$ Franz Volhard Clinic, Charité, HELIOS Clinics, Berlin, Germany.
}

\begin{abstract}
Mutations in genes encoding desmosomal proteins have been identified as the major cause of arrhythmogenic right ventricular dysplasia/cardiomyopathy (ARVC), in which the right ventricle is "replaced" by fibrofatty tissue, resulting in lethal arrhythmias. In this issue of the JCI, Garcia-Gras et al. demonstrate that cardiac-specific loss of the desmosomal protein desmoplakin is sufficient to cause nuclear translocation of plakoglobin, upregulation of adipogenic genes in vitro, and a shift from a cardiomyocyte to an adipocyte cell fate in vivo (see the related article beginning on page 2012). This evidence for potential $\mathrm{Wnt} / \beta$-catenin signaling defects sets the scene for a comprehensive exploration of the contributions of this pathway to the pathophysiology of ARVC, not only through perturbation of cardiac patterning and development, but also through effects on myocardial differentiation and physiology.
\end{abstract}

\section{Unique clinical features of ARVC}

Arrhythmogenic right ventricular dysplasia/cardiomyopathy (ARVC) is a heart muscle disorder that predominantly affects the right ventricle and is associated with ventricular tachycardia, syncope, and sudden death (1). With the prevention of lethal arrhythmia, contractile failure is emerging as a major source of morbidity and mortality. At autopsy there is loss of myocardial mass with "replacement" by abnormal adipose and fibrous tissue; in essence a myocardial dystrophy. These features patchily involve the right ventricle and preferentially affect 3 areas: the apex, the inflow tract, and the outflow tract. This distribution may reflect a primary defect in morphogenesis. Although right ventricular disease predominates, the left ventricle is involved in about $50 \%$ of cases,

Nonstandard abbreviations used: ARVC, arrhythmogenic right ventricular dysplasia/cardiomyopathy; PKP2, plakophilin 2.

Conflict of interest: The authors have declared that no conflict of interest exists.

Citation for this article: J. Clin. Invest. 116:1825-1828 (2006). doi:10.1172/JCI29174. and in some individuals there may be a global dilated cardiomyopathy (1).

\section{Human genetics}

ARVC is typically inherited as a dominant Mendelian disease, although recessive variants exist and the involvement of family members often can only be detected by directed screening (1). Human genetic studies over the last few years have offered insight into the potential causes of ARVC. Early work demonstrated substantial genetic heterogeneity, and at least 9 independent loci have now been identified. The discovery of cutaneous and hair follicle involvement in recessive forms of ARVC led to the identification of mutations in the desmosomal proteins plakoglobin and desmoplakin $(2,3)$. These findings also implicated other desmosomal proteins or their partner proteins as candidate causes of the disorder (Table 1). Subsequent work has revealed desmoplakin mutations in a small proportion of dominantly inherited ARVC cases and in arrhythmogenic cardiomyopathy localized to the left ventricle (4-6). The description of mutations in the cardiac ryanodine receptor in families with an exercise-related arrhythmia known as catecholaminergic polymorphic ventricular tachycardia has highlighted phenotypic distinctions from typical ARVC (1).

In the last year, ARVC genetics have taken a significant step forward, led by the observation that mice null for the armadillo protein plakophilin 2 (PKP2), another desmosomal component, die at around E11 with profound cardiac abnormalities (7). These mice fail to form normal cardiac desmosomes, and desmoplakin dissociates from the abnormal junctions accumulating in cytoplasmic aggregates. These findings led in turn to the discovery of dominant mutations in the PKP2 gene in a large proportion of probands with ARVC and not only established mutant desmosomal proteins as a major cause of the syndrome, but also raised the possibility of genetic testing as a diagnostic tool (8). The initial report in a series of 120 unselected European probands identified PKP2 mutations in almost one-third of these individuals (8). Recent data from more selected cohorts of index patients with evidence of familial involvement have suggested that as many as $70 \%$ of such kindreds may have mutations in PKP2 (9). Of note, these investigators also described evidence of founder effects for several PKP2 mutations in remote kindreds, implying less dramatic effects on survival than are seen in other forms of ARVC.

\section{Possible disease mechanisms}

How do these mutant junctional proteins result in a unique, predominantly right ventricular cardiac phenotype? Desmosomal proteins are widely expressed, so the focal nature of apparent pathology in both 
Table 1

Human cardiac desmosomal diseases

\begin{tabular}{|c|c|c|c|c|c|c|}
\hline Disorder & OMIM no. & Genetic Iocus & Causative gene & Mode of inheritance & Comments & References \\
\hline ARVC8 & $607450 ; 125647$ & $6 p 24$ & Desmoplakin 1 & $A D$ & $\begin{array}{l}\text { Highly pleiotropic, occasionally } \\
\text { involves LV cardiomyopathy }\end{array}$ & 4,6 \\
\hline Carvajal syndrome & 605676 & $6 p 24$ & Desmoplakin 1 & $A R$ & $\begin{array}{l}\text { LV involvement, aneurysm, } \\
\text { palmoplantar keratoderma, } \\
\text { and woolly hair }\end{array}$ & 3 \\
\hline ARVC9 & 602861 & $12 \mathrm{p} 11$ & PKP2 & $A D$ & Present in $30-70 \%$ of cases & 8 \\
\hline Naxos disease & 601214 & $17 q 21$ & Plakoglobin & AR & $\begin{array}{c}\text { Palmoplantar keratoderma } \\
\text { and woolly hair }\end{array}$ & 2 \\
\hline ARVC & 125671 & $18 q 12$ & Desmoglein 2 & $A D$ & & 24 \\
\hline
\end{tabular}

AD, autosomal dominant; AR, autosomal recessive; OMIM, Online Mendelian Inheritance in Man.

dominant and recessive ARVC led to initial speculation on the role of mechanical stresses $(1,10,11)$. Impaired desmosome function under conditions of mechanical stress was proposed to predispose to cardiomyocyte detachment and death, with subsequent inflammatory reaction and fibrofatty replacement. However, consideration of the distribution of skin lesions in recessive variants infers that this mechanism alone is unlikely to be responsible. Several areas of the body subject to substantial physical stresses are not involved, while the hair follicles are uniformly affected. In addition, the prominent adipose replacement suggests not scarring and healing, but rather a more fundamental perturbation of primary tissue architecture.
Three different types of intercellular junction are distinguished at the cardiac intercalated disc: (a) adherens junctions, which anchor actin filaments; (b) desmosomes, which anchor intermediate filaments; and (c) gap junctions, which mediate ion transfer (Figure 1). Cardiac myocytes rely on these specialized structures for both mechanical and electrical

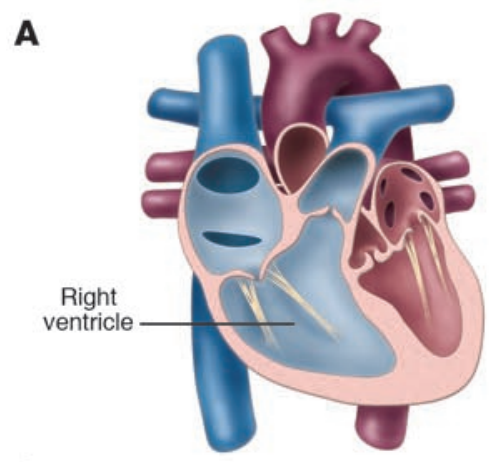

Desmosome

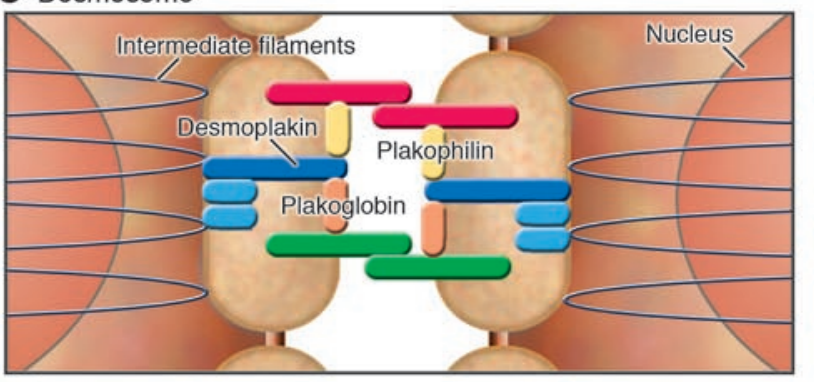

B Cardiac myocytes

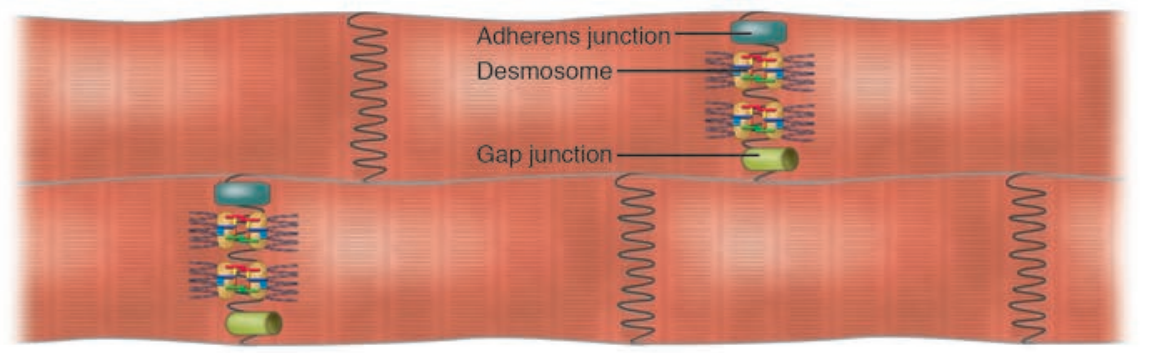

D

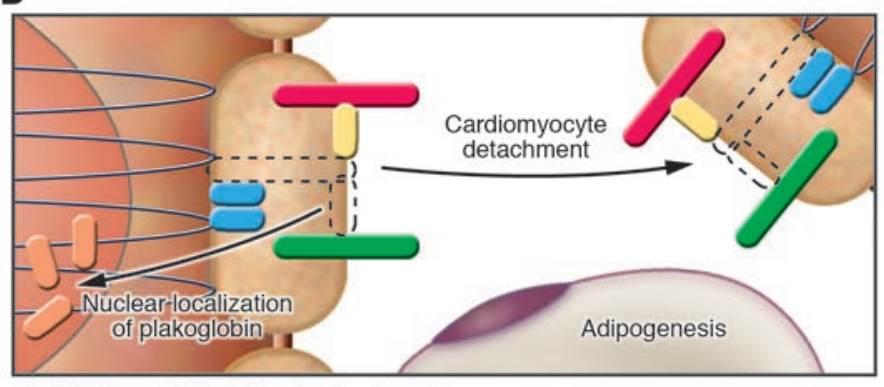

Inhibition of Wnt/B-catenin signaling

Increased number of adipocytes

Increased fibrosis and myocyte apoptosis

Ventricular arrhythmias and contractile dysfunction

\section{Figure 1}

Cardiac-specific restriction of the desmosomal protein desmoplakin causes nuclear localization of plakoglobin and reduced Wnt/3-catenin signaling, recapitulating human ARVC. (A) ARVC predominantly affects the right ventricle of the heart. (B) The intercalated discs of cardiac myocytes are characterized by gap junctions, adherens junctions, and desmosomes. (C) Cell-cell adhesion is largely dependent on interaction of intracellular components of the desmosomal plaque such as desmoplakin and plakoglobin. (D) In this issue of the JCl, Garcia-Gras et al. (16) report that heterozygous cardiac desmoplakin-deficient mice show nuclear localization of plakoglobin and reduced $\mathrm{Wnt} / \beta$-catenin signaling. This causes increased expression of adipogenic and fibrogenic genes in vitro, abnormal cardiac adipose tissue and fibrosis in vivo, and ventricular arrhythmias similar to human ARVC. Interactions between signaling defects and mechanical stresses are likely to be involved in the genesis of the final phenotype. 
coupling of the myocardial syncytium (12). Desmosomes may protect other junctions from mechanical stress, but they also have been implicated in the structural organization of the intercalated disc. Desmosome-dependent orchestration of local membrane and cytoplasmic domains may be critical for many of the physiologic functions of the intercalated disc (12). For example, the destabilization of cell adhesion complexes may perturb the kinetics of gap junction turnover, resulting in heterogeneous conduction, a potential contributor to arrhythmogenesis in $\operatorname{ARVC}(13,14)$.

Desmosomes also participate in intercellular signaling networks, of which the Wnt/ $\beta$-catenin pathway is the most extensively studied (15). In the archetypal pathway the cytoplasmic concentration of $\beta$-catenin is exquisitely regulated by multiple inputs, including secreted ligands of the Wnt family and recruitment of $\beta$-catenin to intercellular junctional complexes. Cytoplasmic accumulation of $\beta$-catenin leads to its nuclear translocation, association with the $\mathrm{T}$ cell factor/lymphoid enhancer factor (Tcf/Lef) family of transcription factors and subsequent changes in gene expression. This evolutionarily conserved pathway plays a central role in many of the most fundamental cellular behaviors and has been directly implicated in the regulation of cell fate, proliferation, and apoptosis. Importantly, the various pathway components are duplicated in higher organisms, and specific isoforms may even be employed serially for discrete functions at different times and at different sites (15). In addition, superimposed on the basic structure of the $\mathrm{Wnt} / \beta$-catenin signaling network are many subtle feedback loops and points of cross-talk that are only beginning to be uncovered (15).

In this context, in this issue of the JCI Garcia-Gras et al. explored the effects of cardiac-restricted desmoplakin deficiency on canonical Wnt/ $\beta$-catenin signaling (16). Using Cre recombinase driven by an $\alpha$-myosin heavy chain gene promoter with a floxed desmoplakin allele, desmoplakin expression was eliminated in the heart. Homozygous cardiac deletion of desmoplakin results in substantial embryonic lethality, with evidence of growth arrest at around E11 and cardiac abnormalities reminiscent of the phenotypes seen with germline desmoplakin-null or PKP2-null mice $(7,17)$. Approximately $5 \%$ of cardiac null desmoplakin homozygotes survive to gestation, but these mice die within 6 weeks. Mice heterozygous for the conditional null allele develop age-dependent multichamber cardiac enlargement and dystrophic myocardium with many of the hallmarks of ARVC, including disorganized myocytes and areas of fibrous and adipose tissue. The authors also convincingly demonstrate - using siRNA in HL-1 cells that inhibition of desmoplakin expression is sufficient to cause nuclear translocation of plakoglobin and upregulation of genes implicated in adipogenesis and generation of new collagen. Increased cardiomyocyte nuclear localization of plakoglobin and ventricular arrhythmias was also observed in heterozygous mice (Figure 1). These results infer that desmoplakin deficiency results in inhibition of canonical Wnt/ $\beta$-catenin signaling and a consequent shift away from a myocyte fate and toward an adipocyte fate in the heart. These data support previous evidence of competition between plakoglobin and $\beta$-catenin and demonstrate that disruption of the Wnt/ $\beta$-catenin axis is sufficient to push cell fate toward adipogenesis, even in myocardium $(15,18)$. Clearly more work will be required to reconcile these data with the proposed mechanisms in ARVC families resulting from mutations in plakoglobin itself (13).

\section{Multiple mechanisms}

It remains to be seen how mechanistically faithful this model will prove to be for human ARVC. Human desmoplakinassociated cardiomyopathy results from germline mutations (3-6), and the effects of germline desmoplakin knockout suggest either that the known disease alleles are unlikely to be true nulls or that the mouse is not a perfect model of human disease (17). The relatively late excision of the floxed allele in relation to cardiogenesis and the restriction of the resultant null allele to the heart preclude an assessment of the effects of perturbed Wnt/ $\beta$-catenin signaling on earlier developmental events that may be central to the pathogenesis of ARVC. Wnt signaling from the neural tube inhibits cardiogenesis in anterior adaxial mesoderm (19), while regional inhibition of Wnt activity is necessary for the normal induction of cardiogenesis in the anterior lateral mesoderm $(20,21)$. Inhibition of Wnt signaling in the posterior mesoderm is sufficient to induce ectopic cardiac tissue, and conditional knockout of $\beta$-catenin in the endoderm results in cardiac duplication in the midline (22). Once the initial heart tube is patterned, restriction of endocardial $\beta$-catenin signaling is critical for normal valve formation (23). In addition to these and other possible roles for the Wnt/ $\beta$-catenin pathway, a direct effect of plakoglobin signaling is also a potential explanation for the observations outlined in the current work (16). The successful dissection of the pathobiology of ARVC will depend on understanding the role of human desmoplakin disease alleles in each of these developmental steps as well as in myocyte proliferation and differentiation and in postnatal physiology.

Garcia-Gras et al. (16) have established a potential role for signaling defects in ARVC, setting the scene for a comprehensive exploration of the interplay of cell adhesion proteins not only as passive players in myocardial architecture, but as key regulators in cardiac patterning and development, in myocyte differentiation, and in the ongoing maintenance of the cellular architecture of the adult heart. Superimposed mechanical stresses may well play a major role in ARVC (14), but the pathogenesis of this disorder is likely to reflect the unique molecular networks responsible for the development and maintenance of the right ventricle. Mouse modeling has taught us that human disease is often the result not of true null alleles, but rather of subtle hypomorphic or gainof-function effects precisely perturbing complex intersecting pathways. Ultimately, understanding the many roles of the Wnt/ $\beta$-catenin pathway in the biology of ARVC will require the generation of a series of tissue-specific conditional null alleles and the recapitulation of disease alleles.

Address correspondence to: Calum A. MacRae, Massachusetts General Hospital, Cardiovascular Research Center, CNY1494218, 149 13th Street, Charlestown, Massachusetts 02129, USA. Phone: (617) 726-4343; Fax: (617) 726-5806; E-mail: macrae@cvrc.mgh.harvard.edu. Or to: Ludwig Thierfelder, Max Delbrueck Center for Molecular Medicine, Robert Rossle Strasse 10, 13092 Berlin, Germany. Phone: 49-309417-2398; Fax: 49-30-9417-2540; E-mail: lthier@mdc-berlin.de.

\footnotetext{
1. Sen-Chowdhry, S., Lowe, M.D., Sporton, S.C., and McKenna, W.J. 2004. Arrhythmogenic right ventricular cardiomyopathy: clinical presentation, diagnosis, and management. Am. J. Med. 117:685-695.

2. McKoy, G., et al. 2000. Identification of a deletion in plakoglobin in arrhythmogenic right ventricular cardiomyopathy with palmoplantar keratoderma and woolly hair (Naxos disease). Lancet. 355:2119-2124.

3. Norgett, E.E., et al. 2000. Recessive mutation in desmoplakin disrupts desmoplakin-intermediate filament interactions and causes dilated cardio-
} 
myopathy, woolly hair and keratoderma. Hum. Mol. Genet. 9:2761-2766.

4. Rampazzo, A., et al. 2002. Mutation in human desmoplakin domain binding to plakoglobin causes a dominant form of arrhythmogenic right ventricular cardiomyopathy. Am. J. Hum. Genet. 71:1200-1206.

5. Bauce, B., et al. 2005. Clinical profile of four families with arrhythmogenic right ventricular cardiomyopathy caused by dominant desmoplakin mutations. Eur. Heart J. 26:1666-1675.

6. Norman, M., et al. 2005. Novel mutation in desmoplakin causes arrhythmogenic left ventricular cardiomyopathy. Circulation. 112:636-642.

7. Grossmann, K.S., et al. 2004. Requirement of plakophilin 2 for heart morphogenesis and cardiac junction formation. J. Cell Biol. 167:149-160.

8. Gerull, B., et al. 2004. Mutations in the desmosomal protein plakophilin-2 are common in arrhythmogenic right ventricular cardiomyopathy. Nat. Genet. 36:1162-1164.

9. van Tintelen, J.P., et al. 2006. Plakophilin-2 mutations are the major determinant of familial arrhythmogenic right ventricular dysplasia/cardiomyopathy. Circulation. 113:1650-1658.

10. Protonotarios, N., et al. 2001. Genotype-phenotype assessment in autosomal recessive arrhythmogenic right ventricular cardiomyopathy (Naxos disease) caused by a deletion in plakoglobin. J. Am. Coll. Cardiol. 38:1477-1484.

11. Alcalai, R., Metzger, S., Rosenheck, S., Meiner, V. and Chajek-Shaul, T. 2003. A recessive mutation in desmoplakin causes arrhythmogenic right ventricular dysplasia, skin disorder, and woolly hair. J. Am. Coll. Cardiol. 42:319-327.

12. Jamora, C., and Fuchs, E. 2002. Intercellular adhesion, signalling and the cytoskeleton. Nat. Cell Biol. 4:E101-E108

13. Kaplan, S.R., et al. 2004. Remodeling of myocyte gap junctions in arrhythmogenic right ventricular cardiomyopathy due to a deletion in plakoglobin (Naxos disease). Heart Rhythm. 1:3-11.

14. Kostetskii, I., et al. 2005. Induced deletion of the $\mathrm{N}$-cadherin gene in the heart leads to dissolution of the intercalated disc structure. Circ. Res. 96:346-354.

15. Huelsken, J., and Birchmeier, W. 2001. New aspects of Wnt signaling pathways in higher vertebrates. Curr. Opin. Genet. Dev. 11:547-553.

16. Garcia-Gras, E., et al. 2006. Suppression of canonical Wnt $/ \beta$-catenin signaling by nuclear plakoglobin recapitualtes phenotype of arrhythmogenic right ventricular cardiomyopathy. J. Clin. Invest.
116:2012-2021. doi:10.1172/JCI27751

17. Gallicano, G.I., et al. 1998. Desmoplakin is required early in development for assembly of desmosomes and cytoskeletal linkage. J. Cell Biol. 143:2009-2022.

18. Ross, S.E., et al. 2000. Inhibition of adipogenesis by Wnt signaling. Science. 289:950-953.

19. Tzahor, E., and Lassar, A.B. 2001. Wnt signals from the neural tube block ectopic cardiogenesis. Genes Dev. 15:255-260.

20. Marvin, M.J., Di Rocco, G., Gardiner, A., Bush, S.M., and Lassar, A.B. 2001. Inhibition of Wnt activity induces heart formation from posterior mesoderm. Genes Dev. 15:316-327.

21. Schneider, V.A., and Mercola, M. 2001. Wnt antagonism initiates cardiogenesis in Xenopus laevis. Genes Dev. 15:304-315.

22. Lickert, H., et al. 2002. Formation of multiple hearts in mice following deletion of beta-catenin in the embryonic endoderm. Dev. Cell. 3:171-181.

23. Hurlstone, A.F., et al. 2003. The Wnt/beta-catenin pathway regulates cardiac valve formation. Nature. 425:633-637.

24. Pilichou, K., et al. 2006. Mutations in desmoglein-2 gene are associated with arrhythmogenic right ventricular cardiomyopathy. Circulation. 113:1171-1179.

\title{
Neuropeptide signaling and hydrocephalus: SCO with the flow
}

\begin{abstract}
David J. Picketts
Molecular Medicine Program, Ottawa Health Research Institute, and Department of Medicine, Department of Biochemistry, Microbiology, and Immunology, and Centre for Neuromuscular Disease, University of Ottawa, Ottawa, Ontario, Canada.
\end{abstract}

Congenital hydrocephalus affects $0.1-0.3 \%$ of live births, with a high mortality rate $(-50 \%)$ in the absence of surgical intervention. Although the insertion of shunts alleviates the symptoms of the majority of congenital cases, the molecular basis of hydrocephalus and the mechanisms of cerebrospinal fluid (CSF) circulation remain largely unknown. Two important players are the subcommissural organ/Reissner's fiber (SCO/RF) complex and the ventricular ependymal (vel) cells that together facilitate the flow of the CSF through the narrow canals of the ventricular system. In this issue of the JCI, Lang et al. demonstrate that overexpression of the pituitary adenylate cyclase-activating polypeptide (PACAP) type I (PAC1) receptor gene results in abnormal development of the $\mathrm{SCO}$ and vel cells, leading to congenital hydrocephalus (see the related article beginning on page 1924). The ligand for the PAC1 receptor is the neuropeptide PACAP, which uncovers what the authors believe to be a novel role for this signaling cascade in the regulation of CSF circulation.

Hydrocephalus arises from an accumulation of cerebrospinal fluid (CSF), most

Nonstandard abbreviations used: CREB, cAMP response element-binding protein; $\mathrm{CSF}$, cerebrospinal fluid; IFT, intraflagellar transport; PAC1, PACAP type I; PACAP, pituitary adenylate cyclase-activating polypeptide; RF, Reissner's fiber; SCO, subcommissural organ; vel, ventricular ependymal.

Conflict of interest: The author has declared that no conflict of interest exists.

Citation for this article: J. Clin. Invest. 116:1828-1832 (2006). doi:10.1172/JCI29148. frequently due to an impairment of CSF flow within either the ventricular system (noncommunicating hydrocephalus) or the subarachnoid space (communicating hydrocephalus). The CSF is secreted from the choroid plexus, and movement through the ventricular system occurs in a rostrocaudal direction, from the lateral ventricles to the third ventricle via the foramen of Munro, then through the Sylvian aqueduct to the fourth ventricle, and finally into the cisterna magna of the sub- arachnoid space and the central canal of the spinal cord (Figure 1). Ultimately, CSF fluid is removed through the arachnoid villi into the venous circulation.

Noncommunicating hydrocephalus results from an obstruction of the ventricular system and has many causes, including viral infection, tumors, hemorrhage, and developmental defects (1). Obstruction usually occurs in the narrowed segments of the ventricular system, typically the cerebral aqueduct. Indeed, stenosis of the cerebral aqueduct is considered the primary cause of congenital hydrocephalus $(1,2)$. Several factors play a role in the maintenance of CSF flow through the narrow canals, including ciliary movement on ependymal cells and a functioning subcommissural organ (SCO), an ependymal gland located in the dorsocaudal region of the third ventricle at the entrance of the Sylvian aqueduct (Figure 2$)(3,4)$. It is well established that the SCO secretes glycoproteins that aggregate and form a long, threadlike structure known as Reissner's fiber (RF) in most vertebrate species (Figure 2). RF elongates and extends through 